Der Wallstein-Preis 2009 wurde Frau Susanne Krones, München, für ihr Werk „Akzente im Carl Hanser Verlag. Geschichte, Programm und Funktionswandel einer Literarischen Zeitschrift" verliehen.

\title{
„Akzente“ im Carl Hanser Verlag. Geschichte, Programm und Funktionswandel einer literarischen Zeitschrift
}

\section{SusAnne Krones}

Wer über gegenwärtige Akteure des deutschen Literaturbetriebs wie den Carl Hanser Verlag, die dort erscheinende literarische Zeitschrift Akzente und ihre drei Herausgeber - den 2003 verstorbenen Literaturwissenschaftler Walter Höllerer, den Nachkriegsautor Hans Bender und den heutigen Hanser-Verleger, AkzenteHerausgeber und Schriftsteller Michael Krüger - forscht, trifft zwangsläufig auf ein zeitgemäßes Meinungsbild: Der Carl Hanser Verlag ist heute bekannt als deutscher Verlag weltberühmter Autoren wie Italo Calvino, Umberto Eco, Milan Kundera, Harry Mulisch, T. C. Boyle, Philip Roth oder Susan Sontag, als Verlag zahlreicher Nobelpreisträger, darunter Ivo

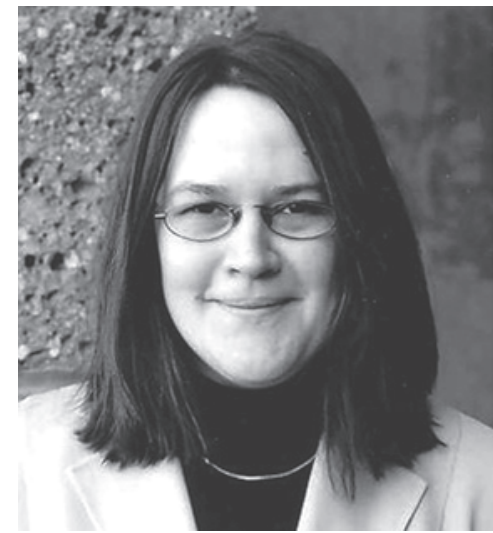

Susanne Krones, promovierte Literatur- und Buchwissenschaftlerin, Lehrbeauftragte an der LudwigMaximilians-Universität München, der Universität Regensburg sowie Verlagslektorin beim Deutschen Taschenbuch Verlag, Trägerin des Wallstein-Preises 2009 Andriæ, Elias Canetti, Joseph Brodsky, Orhan Pamuk und jüngst Herta Müller. Umgekehrt hat der Verlag deutsche Autoren wie etwa Botho Strauß und Günter Kunert weltweit etabliert. Hanser gilt spätestens seit den programmatischen, personellen bzw. ökonomischen Krisen der Häuser Suhrkamp und Fischer neben Piper und Rowohlt als der Verlag der Gegenwartsliteratur - der deutschen wie der internationalen in deutscher Übersetzung. ${ }^{1}$

$1 \quad$ Vgl. auch Susanne Krones: „Der Warencharakter des Buches wird weiter zunehmen«. Interview mit Edda Ziegler", in: FORUM 2/2004, S. 62f., und Edda Ziegler: 100 Jahre Piper. Die Geschichte eines Verlags. München/Zürich: Piper 2004. 
Im Vergleich zu ihrem Verlag ist die 1954 gegründete Zeitschrift Akzente heute ein leises Forum bedeutender Literatur: Namentlich zwar bekannt, rezipiert aber nur mehr von Insidern in einer kleinen Auflage von rund 3200 Exemplaren, teilt die Zeitschrift das Schicksal ihrer Artgenossen und zeigt sich doch ungewöhnlich robust: 2004 konnten die Akzente ihren 50. Geburtstag feiern und erscheinen auch noch 2010, im 57. Jahrgang nichts Selbstverständliches für etwas so Fragiles, weil Zeitgebundenes und Unrentables, wie eine literarische Zeitschrift.

Dass von den Akzenten ein Zauber ausgeht, der auch international trägt, eine offensichtliche Notwendigkeit, belegt die Art und Weise, wie sich Autoren in den verschiedensten Kontexten über diese Zeitschrift äußern. Der Exilautor Hermann Kesten konstatierte: „Ich habe eben die Odyssee gelesen, von A-Z, nun lese ich die Ilias, wenn meine Ferien länger wären, so wäre es ein hübscher Anfang, um nochmals die Weltliteratur Revue passieren zu lassen, enden müßte ich jedenfalls mit Akzente". ${ }^{2}$ Dererlei Stimmen ließen sich viele zitieren, sie beschreiben die Akzente als "Zentralorgan des Museums der modernen Poesie“ oder als „umfangreichste Anthologie der internationalen Poesie in Deutschland“. Was sie nicht verrieten: welche Häutungen die Zeitschrift durchgemacht hat in ihrer fünfzigjährigen Geschichte und wie sie funktioniert - die Poesiemaschine Akzente.

\section{Fragestellung und Methodik}

Ziel meines Dissertationsprojekts war es, die wechselhafte Geschichte der Literaturzeitschrift Akzente von ihrer Gründung 1954 bis zu ihrem fünfzigsten Jahrgang, 2003, vorzulegen, diese Geschichte in einen Kontext zu setzen mit der Geschichte des Carl Hanser Verlags sowie vorausgehenden oder parallel erscheinenden Literaturzeitschriften wie Corona, Konturen, Texte und Zeichen sowie Höllerers zweiter Zeitschriftengründung Sprache im technischen Zeitalter. Das Programm der Akzente - immerhin 300 Ausgaben mit literarischen Beiträgen, insgesamt 4235 epischen, lyrischen, dramatischen und essayistischen Texten - sowie übergreifende Entwicklungszusammenhänge der literarischen Produktion wie der Rezeption im Umfeld der Akzente sollten dargestellt werden, um so einerseits der literaturgeschichtlichen Bedeutung der Akzente nachzufragen, andererseits auch generell den veränderten Bedingungen für literarische Zeitschriften und dem damit verbundenen Wandel ihrer Funktionen seit den 1950er Jahren nachzuspüren.

2 Volker Neuhaus: „Porträt im Spiegel. Zu den Briefen an Hans Bender“, in: Briefe an Hans Bender, hrsg. v. Volker Neuhaus. München: Hanser 1984, S. 9 
Nach dem Entwurf einer Theorie der literarischen Zeitschrift, die helfen soll, das umfangreiche Quellenmaterial mit einigen grundlegenden Fragestellungen zu systematisieren, und die im Wesentlichen auf systemtheoretischen und netzwerkanalytischen Grundlagen beruht, wird die Geschichte der Akzente als eine Institutionsgeschichte rekonstruiert. Da eine Literaturgeschichte der Akzente jeden Rahmen gesprengt hätte, lag der Schwerpunkt der Recherchen auf der Verlagskorrespondenz, also den Briefwechseln zwischen den Akzente-Herausgebern und ihren Kollegen in Verlagsleitung, Lektorat, Herstellung und Marketing des Carl Hanser Verlags. Die Autorenkorrespondenzen wurden punktuell zur Ergänzung herangezogen. Periodisiert wird die Geschichte der Akzente in Vorgeschichte, Gründungsphase sowie die Herausgeberphasen Walter Höllerer und Hans Bender (19541967), Hans Bender (1968-1976), Hans Bender und Michael Krüger (1976-1980) und Michael Krüger (seit 1981). Der historische Überblick endet mit Ausführungen zum Wandel der wirtschaftlichen, technischen und organisatorischen Rahmenbedingungen.

In einem nächsten Schritt galt es, das Programm der Akzente, verstanden im Sinn eines Verlagsprogramms als das Textkorpus, das sich im Lauf von fünfzig Jahren Zeitschriftengeschichte gebildet hat, auszuwerten - nach literarischen Genres, dem urheberrechtlichen Status der Texte, nach der Komposition der Hefte und ihren festen Rubriken und Strukturprinzipien. Ergänzt wurden die Ausführungen zum Programm der Akzente um Angaben zum Autorenstamm der Zeitschrift und zu dem nationalen und internationalen Netzwerk aus Buch- und Zeitschriftenverlagen, in das die Akzente eingebunden sind, sowie zu fremdsprachigen Literaturen und deutschdeutschen Literaturbeziehungen in den Akzenten.

Im letzten Schritt schließlich wird der Wandel in Funktionen und Selbstverständnis der Zeitschrift an drei Themenkomplexen vorgeführt: Untersucht wurden die Programmatik der drei Herausgeber, die Grenze zwischen Programmatik und Praxis sowie der Wandel von Buchmarkt, Literaturbetrieb und, damit verbunden, der Herstellung literarischer Öffentlichkeit im Laufe der Geschichte der Zeitschrift.

Die Arbeit ist als interdisziplinäre Untersuchung angelegt, die sowohl literatur-als auch buchwissenschaftliche Fragestellungen zu klären versucht. Die literaturhistorische Relevanz ihres Gegenstands zeigt bereits eine kursorische Durchsicht der über 300 bisher erschienenen Hefte: Die Akzente, im Untertitel zunächst als Zeitschrift für Dichtung, später als Zeitschrift für Literatur bezeichnet, verstanden sich selbst als Zeitschrift ohne Programm und als Plattform für die Förderung junger Autoren. Hans Magnus Enzensberger etwa debütierte in den Akzenten, Günter Grass publizierte dort als 
junger Autor und wurde in der Folge wesentlich von Walter Höllerer gefördert, Ingeborg Bachmann war seit der Gründung der Zeitschrift AkzenteAutorin. Undogmatisch und reichhaltig, ist die Literaturzeitschrift zu einem Kreuzungspunkt der Literaturproduktion und der Literaturvermittlung gerade in der Adenauerzeit geworden: Das Spektrum der Akzente-Autoren ist weit und reicht von den Vertretern einer „inneren Emigration“ über die Exilanten und die Mitglieder der Gruppe 47 bis hin zu Vertretern radikaler ästhetischer und literaturtheoretischer Positionen. Außerdem spielten die Akzente eine zentrale Rolle für die Vermittlung ausländischer Literatur in der jungen Bundesrepublik. Aus buchwissenschaftlicher Perspektive ist das Quellenmaterial von großem Interesse, weil es eine intensive Auseinandersetzung mit der konkreten Herausgebertätigkeit von Höllerer und Bender und der Kooperation der Herausgeber mit dem Verlag erlaubt und so einen Blick „hinter die Kulissen“ gewährt. Die Auswertung bietet so einen Baustein für eine „Alltagsgeschichte“ der literarischen Vermittlung im 20. Jahrhundert - und leistet damit einen Beitrag zur Funktionsbestimmung literarischer Zeitschriften. Die literatur- und die buchwissenschaftliche Perspektive treffen sich an dem Punkt, an dem es um die Funktion literarischer Zeitschriften geht.

Dass bisher derart wenig Forschungsliteratur zu literarischen Zeitschriften existiert, ist irritierend, weil deren Rolle als zentrale Funktionsträger literarischer und politischer Entwicklungen, Medien neuer Trends und Tendenzen, Indikatoren der Veränderungen im Publikumsgeschmack ebenso wie Überlieferungsträger der Sozialisationsprozesse weithin anerkannt ist. ${ }^{3}$ Für die Akzente sind die umfangreichen Archivbestände bisher nicht systematisch aufgearbeitet worden.

\section{Quellen}

Wer in einem Verlag arbeitet, muß, wenn er nicht gerade telefoniert, liest oder spricht, schreiben: Aktennotizen, Briefe, Gutachten, Klappentexte, Zeugnisse, Reden zur Weihnachtsfeier. [...] Ein Lebenswerk aus Nebensächlichkeiten. ${ }^{4}$ Genau diese Bestandteile sind es, Überbleibsel alltäglicher Verlagsarbeit, die Michael Krüger als Lebenswerk aus Nebensächlichkeiten beschreibt, aus denen die Akzente-Korrespondenz besteht. Die Briefwechsel zwischen den Akzente-Herausgebern und ihren Autoren sowie ihren

3 Alfred Estermann: „Deutsche Literatur-Zeitschriften 1880-1918“, in: Estermann, Alfred: Kontextverarbeitung. Buchwissenschaftliche Studien. München: K. G. Saur 1998, S. 143

4 Michael Krüger im Vorwort zu seinem Band Vorreden, Zwischenworte, Nachrufe. Ein (lückenhaftes) $A B C$. München/Wien: Sanssouci 2003, S. 7 
Kollegen im Carl Hanser Verlag geben Einblick in die Verlagsarbeit, in Abstimmungsprozesse zwischen Verlag und Redaktion, in die interne Kommunikation und Struktur des Netzwerks um Walter Höllerer, Hans Bender und später Michael Krüger. Ihre Auswertung ist die wesentliche Grundlage der vorliegenden Arbeit.

Der erste Teil der Akzente-Korrespondenz liegt in dem von Walter Höllerer 1977 gegründeten Literaturarchiv Sulzbach-Rosenberg. Dieser Briefwechsel umfasst etwa 35.000 Briefe zwischen Autoren und Redaktion sowie zwischen Redaktion und Verlag im Zeitraum 1952-1970, außerdem Manuskripte, Typoskripte und Druckfahnen. Ihr zweiter Teil war bis zu dessen Einsturz Bestand des Historischen Archivs der Stadt Köln. Der dortige Vorlass von Hans Bender umfasst insgesamt 35.762 Blatt, bestehend aus der Fortsetzung der Akzente-Korrespondenz mit den Autoren und dem Verlag (11.253 Blatt) sowie Einzelbriefwechseln, privater Korrespondenz, Unterlagen zu Akademien und zum P.E.N., zu Benders Anthologien und zur Zeitschrift Jahresring. Die bis 1980 bereits in öffentlichen Archiven zugängliche Akzente-Korrespondenz findet ihre Fortsetzung in ihrem dritten Teil im Verlagsarchiv des Carl Hanser Verlags.

\section{„Wege durch unsicheres Gelände“. Die Geschichte der Akzente}

Lässt man die Geschichte der Zeitschrift noch einmal Revue passieren, zeigen sich die folgenden Entwicklungsschritte als die wesentlichen: Die Vorgeschichte der Zeitschrift (bis 1953) entfaltete das Panorama, in das hinein die Literaturzeitschrift Akzente gegründet wurde - die bis zu Höllerers Lyrikband Der andere Gast komplette Absenz junger, deutschsprachiger Gegenwartsliteratur ließ bereits die Lücke ahnen, in die die Akzente schließlich stießen, und macht das Innovationspotenzial deutlich, das die Zeitschrift in den Verlag eingebracht hat. Hanser hatte vor dem Zweiten Weltkrieg nur wenige belletristische Titel verlegt und die belletristische Produktion während des Krieges schließlich ganz eingestellt; der Fachverlag existierte weiter. Neben der minimalen Backlist aus den Vorkriegsjahren startete Hanser nach 1945 mit Klassikerausgaben und einigen wenigen deutschsprachigen Autoren, Eugen Roth und Emil Strauß, der mit seinem Roman Lebenstanz 1940 ganz auf die Linie nationalsozialistischer Blutund-Boden-Literatur gerückt war. Hanser legte diesen (ursprünglich bei Langen Müller erschienenen) Roman noch 1954 in einer Neuauflage vor.

Die Ausführungen zur Gründungsphase zeigten bereits in nuce, mit welchen Problemen die Akzente-Redaktion im Lauf der folgenden Jahrzehnte 
immer wieder zu kämpfen hatte. Das Ringen um die Konzeption der Zeitschrift, wie es die Jahre 1952/53 prägte, setzte sich fort: Ihre Autonomie in inhaltlichen Fragen mussten die freiberuflichen Herausgeber Walter Höllerer und Hans Bender im alltäglichen Kräftemessen mit ihrem die Zeitschrift finanzierenden Verleger Carl Hanser und seinem Cheflektor Herbert G. Göpfert - beide eine Generation älter - immer neu verteidigen. Unter der Herausgeberschaft Walter Höllerers und Hans Benders entwickelte sich die Zeitschrift von 1954 bis 1967 zu einer der führenden literarischen Zeitschriften der Bundesrepublik. Die Zeitschrift erwies sich als flexibel genug, um zur Integration von literarischen Stimmen aus Innerer Emigration, Exil und der Nachkriegsgeneration beizutragen und um eine Öffnung zur internationalen Literatur gegenüber dem Verlag durchzusetzen und dann mit erstaunlicher Kenntnis und Sensibilität für sich ankündigende Strömungen zu realisieren - eine Entwicklung, die in dieser Dynamik nicht zu prognostizieren war, bedenkt man die geschilderte konservative Ausrichtung des Buchverlages in diesen frühen Jahren. Auf diese Weise konstituierten sich die Akzente erfolgreich im literarischen Feld und bestanden in der Konkurrenz mit Texte und Zeichen, Kursbuch und anderen Zeitschriften. Es war auch das in der Konstruktion der Zeitschrift angelegte und durch die Brillanz, das Renommee und die gute Zusammenarbeit der beiden Gründungsherausgeber beinahe gleichberechtigte Kräftemessen zwischen Zeitschriftenredaktion und Verlag, das zu guten und konkurrenzfähigen Ausgaben führte. Erfolgreiche Synergieeffekte zwischen Zeitschrift und Verlag (wie Höllerers langlebige Reihe Literatur als Kunst) und gescheiterte (wie der zweimalige Versuch, Hans Bender als Lektor an den Carl Hanser Verlag zu binden) belegen, wie eng die Zusammenarbeit beider Partner war. Die für das Verlagswesen als in der Durchdringungszone von Kultur und Wirtschaft angesiedelte Sphäre typische, unlösbare Wechselwirkung zwischen wirtschaftlichen und kulturellen Strategien zeigte sich bei der Einführung von Themenheften und der Forcierung ästhetischer Debatten: Die Zeitschrift war finanziell in eine Krise geraten und stieß nicht mehr auf angemessene Resonanz beim Publikum - von einer veränderten redaktionellen Arbeit versprach man sich höhere Auflagen und durch verstärkte Presseberichterstattung über die Themenhefte auch mehr Renommee.

Walter Höllerer verließ die Zeitschrift schließlich zum Jahreswechsel 1967/68; Hans Bender startete mit den Akzenten als Alleinherausgeber in das Epochenjahr. Einer längst vollzogenen Weitung des Literaturbegriffs gab er mit der Veränderung des Untertitels von Zeitschrift für Dichtung in Zeitschrift für Literatur Ausdruck. Der zunehmende Funktionsverlust literarischer Zeitschriften lässt sich indes schon ahnen: Die Werbung für die 
Zeitschrift muss intensiviert werden, die Themenhefte, die bisher Themenschwerpunkte hatten, verwandeln sich richtiggehend in Anthologien, die sich ganz einem Thema widmen und, ähnlich wie Buchpublikationen, eine je eigene Zielgruppe ansprechen. Die 1975 vollzogene Verkleinerung des Formats der Zeitschrift auf Taschenbuchgröße zeigt die massiven ökonomischen Zwänge und ist als eine Rationalisierungsmaßnahme des Verlages zu verstehen, der die Zeitschrift halten wollte. Zuletzt prägten Innovationen im Satzverfahren die Geschichte der Akzente: Anfangs im Bleisatz gesetzt, dann im Composersatz, bedeutete schließlich der Wechsel zu digitalen Satzverfahren ganz andere Rahmenbedingungen für die Redaktionsarbeit. Der Skandal um das Memorandum der Akademie für Sprache und Dichtung in Darmstadt und deren eigene Zeitschriftenpläne zeigten die Grenzen staatlicher Einflussnahme im Literaturbetrieb, der Reprint bei Zweitausendeins wiederum bewies, das auch für Zeitschriften lukrative Zweitverwertungen möglich sind. Zudem belegte der erstaunliche Erfolg dieses Nachdrucks das Interesse an der Zeitschrift, das bis Anfang der achtziger Jahre ungebrochen war, und das Bedürfnis vieler Leser nach einem Archiv moderner Poesie, wie die Akzente es geschaffen haben.

Der Einstieg des Verlagslektors Michael Krügers als Mitherausgeber in die Zeitschrift ist rückblickend der Auftakt einer gut vierjährigen Übergangszeit (1976-1980), in der die Akzente sich wesentlich veränderten: Schon rein äußerlich verkehrte sich die Konstellation ins Gegenteil. Von einer Zeitschrift mit zwei freiberuflichen Herausgebern wurden die $A k$ zente zu einer Zeitschrift, die fest in Verlagshänden war und von jetzt an eng von Michael Krügers Persönlichkeit getragen wurde. Tatsächlich erwies sich Krüger als Akquisetalent, seine Lektorenerfahrung und die gute Beziehung zwischen Krüger und Bender zahlten sich aus. Schnell merkte Krüger, dass die Perspektive des Herausgebers ganz andere Sehweisen erlaubt als die des zuständigen Lektors im Verlag, die bisher die seine gewesen war. Kaum im Amt, begehrte Krüger gegen die Bedingungen auf, unter denen die Akzente zu dieser Zeit agierten, und konnte dies als Mitarbeiter des Verlages anders, vehementer, tun, als der freie Herausgeber Bender es vermocht hätte. Für die Zeitschrift hatte Krüger das Gefühl, die Herausgeber kämpften alleine gegen die wachsende Konkurrenz, während der Verlag die Autorenhonorare einfror, obwohl diese wesentlich dazu beitragen, eine Zeitschrift konkurrenzfähig zu machen. Seit 1981 verantwortet Michael Krüger die Zeitschrift nun alleine. Er startete in den goldenen Zeiten des Literaturverlages und konnte bei Amtsantritt bereits die schon etwas länger geplante Edition Akzente in Ergänzung zu der Zeitschrift realisieren, deren Programm bis heute vor allem allen Liebhabern des Essays wie eine 
Schublade voller blankgeputzter Murmeln entgegenglitzert. 1986 wurde Michael Krüger Verleger des Carl Hanser Verlages und damit erstmals ein Akzente-Herausgeber zu seinem eigenen Verlagschef.

Die Situation ist paradox: Michael Krüger bündelt als Alleinherrscher und Verleger zwar ungleich mehr Kompetenzen als seine beiden Vorgänger, doch die Zeitschrift selbst ist machtlos geworden - und stiller. Das Nischendasein eröffnet neuen Spielraum für die Programmpolitik: Die Ausrichtung am literarischen Markt verliert an Bedeutung. Krüger kann seinen internationalen Kurs also ohne Bedenken fortsetzen und auch Gewässer ansteuern, für die die Seekarten das gefürchtete „Untiefen!“ melden, in die hineinzufahren sich aber dennoch lohnt, weil das Wasser von unvergleichlichem dunklen Türkis ist. So führt er sein Archiv für internationale Poesie konsequent und mit sicherem Qualitätsbewusstsein weiter, allerdings auch in dem Wissen darum, dass die Zeitschrift dadurch esoterischer anmutet und als entbehrlicher empfunden wird denn in früheren Zeiten. Ganz unschuldig ist sie nicht daran: Wo einst Lyrik von Günter Grass oder Wolf Biermann dezidierten Zeitbezug ins Blatt brachte, fanden die weltpolitischen Ereignisse der achtziger und der neunziger Jahre - ob Mauerfall, Krieg in Jugoslawien oder im Irak oder die Bedrohung durch den internationalen Terrorismus - höchstens in wenigen Zeilen der Herausgebervorworte Eingang in die Zeitschrift.

Für den Verlag ist die Zeitschrift nach wie vor ein wertvolles Label, das für Qualität steht und Autorenpflege ermöglicht. Mit Gastherausgebern füllt Krüger die Lücke, die das Fehlen eines Mitherausgebers lässt, und holt sich je nach Themen oder Sprachraum Spezialistenwissen und andere Netzwerke ins Blatt. Es mutet in der für klassische Literaturzeitschriften scheinbar ausweglosen Situation programmatisch an, dass Krüger seine Zeitschrift mit Les Murrays Gedicht „Gleichmütigkeit“ ins neue Jahrtausend ziehen lässt: mit Gleichmütigkeit im besten Sinn, die die Ruhe bewahrt und das Beste aus der jeweiligen Situation macht. Ein Literaturblatt, das weniger wahrgenommen wird, kann sich mehr erlauben, Verwegenes gar: das Beste.

Das diachrone Kapitel zum Programm der Akzente kann zeigen, dass die Herausgeber in der Komposition der Hefte bestimmte Standards etablierten und förderten, etwa die zwingende Kombination eines Autorenporträts mit literarischen Texten des jeweiligen Autors, auch das Arrangement von Gedichten zu Zyklen. Dass bestimmte Autoren ausschließlich unter einem Herausgeber vorkommen (man denke an Günter Grass zur Herausgeberzeit Walter Höllerers), zeigt dessen Subjektivität. Und die muss sein, steht doch ein Herausgeber ohne Institution für sich, ist auf sein eigenes Netzwerk und seinen eigenen Spürsinn angewiesen. Auch die Zeitschrift selbst ist in 
ein Netzwerk von nationalen und internationalen Buch- und Zeitschriftenverlagen eingebunden, die als Konkurrenten und Partner interagieren. Das Kapitel zur fremdsprachigen Literatur konnte zeigen, wie die Akzente den Nouveau Roman durchsetzten, als eine der ersten in der Bundesrepublik, die Beat-Lyrik vorstellten und den tschechischen Poetismus zumindest mit beförderten. Dass einem in jüngerer Zeit weniger Beispiele einfallen, in denen Akzente junge Strömungen mitbeförderten, zeigt Mehrfaches: einmal, dass Stile an sich unwichtiger werden, was man auch daran beobachten kann, dass selbst eine renommierte Instanz der Literaturförderung wie das Literarische Colloquium Berlin nicht mehr alle geförderten Autoren erfolgreich an einen Verlag vermitteln kann, dass Themen, Plots und das Potenzial, das Autoren als öffentliche Figuren mitbringen, wichtiger für Akquiseentscheidungen werden. Es zeigt aber auch einen Generationswandel an, der sich mit den Akzenten vollzogen hat: Gestartet als attraktives Blatt für die jüngste und die mittlere Generation der Produzenten und Rezipienten von Gegenwartsliteratur, ist die Zielgruppe mit ihrer Zeitschrift älter geworden; die Zielgruppe hat sich etabliert, die Zeitschrift den Glanz einer Entdeckerzeitschrift verloren. Dafür sind andere Zeitschriften nachgewachsen, die sich heute ans Entdecken machen - Zeitschriften sind eben immer auch Angelegenheiten von Generationen.

Zur Zukunft literarischer Zeitschriften in der veränderten literarischen Öffentlichkeit freilich lässt sich noch nichts Verbindliches sagen. Anders als seine Kollegen und im Geist seiner Vorgänger Walter Höllerer und Hans Bender, ist Michael Krüger von zurückhaltendem, doch anhaltendem Optimismus für das Medium und glaubt an eine Renaissance der kleinen Literaturzeitschriften: „Sie sind, wie das Schreibheft und die manuskripte und viele andere, einer winzigen Elite - die es ablehnen wird, sich so zu nennen - vorbehalten, die hartnäckig daran festhält, ihren Weg durch unsicheres Gelände zu gehen. Seltsame Menschen, die sich bei einem Gedicht aufhalten. Einzelgänger, die die Angebote der Wissensgesellschaft für einen Moment in den Wind schlagen. Sonderlinge, denen die Schönheit von ein paar gefügten Worten in die Haut fährt. Es werden immer weniger, dar-

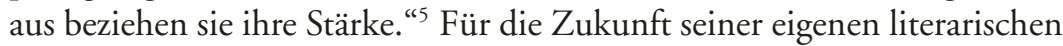
Zeitschrift über Krügers Zeit als Verleger des Carl Hanser Verlages hinaus heißt das freilich: alles und nichts. ${ }^{6}$

5 Michael Krüger: „An die Akzente-Leser“, in: Akzente 1/2000, S. 1f.

6 Susanne Krones' mit dem Wallstein-Preis ausgezeichnete Monographie Akzente im Carl Hanser Verlag. Geschichte, Programm und Funktionswandel einer literarischen Zeitschrift ist 2009 im Wallstein Verlag erschienen (ISBN 978-3-8353-0551-9). 